\title{
Generation of genetically tailored porcine liver cancer cells by CRISPR/Cas9 editing
}

Lobna Elkhadragy' (iD, Maureen R Regan ${ }^{2}$, William M Totura', Kimia Dasteh Goli' ${ }^{1}$, Shovik Patel ${ }^{3}$, Kelly Garcia ${ }^{4}$, Matthew Stewart ${ }^{5}$, Lawrence B Schook ${ }^{1,3,6}$, Ron C Gaba ${ }^{1,7}$ \& Kyle M Schachtschneider*,1,2,6

${ }^{1}$ Department of Radiology, University of Illinois at Chicago, Chicago, IL 60612, USA; ${ }^{2}$ Department of Biochemistry \& Molecular Genetics, University of Illinois at Chicago, Chicago, IL 60607, USA; ${ }^{3}$ Department of Animal Sciences, University of Illinois at Urbana-Champaign, Urbana, IL 61801, USA; ${ }^{4}$ Biological Research Laboratory, University of Illinois at Chicago, Chicago, IL 60612, USA; ${ }^{5}$ Department of Veterinary Clinical Medicine, University of Illinois at Urbana-Champaign, Urbana, IL 61802, USA; ${ }^{2}$ National Center for Supercomputing Applications, University of Illinois at Urbana-Champaign, Urbana, IL 61801, USA; ${ }^{7}$ Department of Pathology, University of IIlinois at Chicago, Chicago, IL 60612, USA; *Author for correspondence: kschach2@uic.edu

BioTechniques 70: 37-48 (January 2021) 10.2144/btn-2020-0119

First draft submitted: 30 July 2020; Accepted for publication: 1 October 2020; Published online: 23 November 2020

\section{ABSTRACT}

Pigs provide a valuable large animal model for several diseases due to their similarity with humans in anatomy, physiology, genetics and drug metabolism. We recently generated a porcine model for $T P 53^{R 167 H}$ and $K R A S^{G 12 D}$ driven hepatocellular carcinoma (HCC) by autologous liver implantation. Here we describe a streamlined approach for developing genetically tailored porcine HCC cells by CRISPR/Cas 9 gene editing and isolation of homogenous genetically validated cell clones. The combination of CRISPR/Cas 9 editing of HCC cells described herein with the orthotopic HCC model enables development of various porcine HCC models, each with a specific mutational profile. This allows modeling the effect of different driver mutation combinations on tumor progression and in vivo testing of novel targeted therapeutic approaches in a clinically relevant large animal model.

\section{METHOD SUMMARY}

Here we describe a streamlined approach for developing genetically tailored porcine cancer cells. We demonstrate high-efficiency CRISPR/Cas9mediated gene editing in porcine hepatocellular carcinoma cells and isolation of homogenous gene knockout clones. These genetically tailored cells can be combined with previously described implantation techniques to develop precision porcine models of hepatocellular carcinoma.

\section{KEYWORDS:}

CRISPR/Cas9 • gene editing • gene knockout • hepatocellular carcinoma $\bullet$ large animal model $\bullet$ liver cancer $\bullet$ porcine cells

Preclinical animal models are crucial tools for testing the safety and efficacy of novel therapeutic approaches prior to human testing. Pig models have been successfully developed and utilized in various fields of research, including cancer, cardiovascular diseases, neurodegenerative diseases, GI tract diseases and stem cell studies [1-3]. A major advantage of pig models is their large size, which enables the use and testing of surgical procedures similar to those used for human patients. Furthermore, pigs share many similarities with humans, including similarities in anatomy, immunity, genetics, drug metabolism and body metabolic rate, which increases the translatability of studies done using porcine models into clinical success [2,3]. We have recently generated a porcine model for hepatocellular carcinoma (HCC) $[4,5]$. HCC, the most common primary liver cancer, is an aggressive disease with poor response to treatment at advanced stages [6]. We developed the HCC model using a transgenic pig, the Oncopig Cancer Model, which harbors Cre recombinase-inducible TP53 ${ }^{R 167 H}$ and $K R A S^{G 12 D}$ transgenes [7]. Porcine HCC cells were developed from Oncopigs by isolating hepatocytes and inducing transgene expression using Cre recombinase. The developed porcine HCC cells were used for implantation into the liver of the same Oncopig, resulting in intrahepatic tumor growth [4,5]. Building on the success of our recent model, we aimed to expand our approach by introducing driver mutations that occur frequently in human HCC to develop genetically tailored HCC tumors. As such, these novel porcine HCC models can be used to investigate the effect of different mutational profiles on tumor progression and chemotherapeutic susceptibility.

The clustered regularly interspaced short palindromic repeats (CRISPR) system has recently emerged as a powerful and effective tool that can introduce genetic mutations in diverse cell types and organisms, including pigs [8,9]. CRISPR systems have two components: guide RNA (gRNA) and a CRISPR-associated (Cas) endonuclease such as Cas9 [8]. The gRNA is composed of CRISPR RNA (crRNA), a 17-20-nucleotide spacer that defines the genomic target to be modified, and a scaffold sequence known as transactivating crRNA (tracrRNA), which is necessary for Cas binding. The Cas endonuclease and the gRNA form a ribonucleoprotein (RNP) complex, which is directed by the gRNA to the genomic target site adjacent to a protospacer-adjacent motif (PAM). Then the endonuclease cleaves the DNA 3-4 nucleotides upstream of the PAM sequence. The resulting double-strand break is predominantly repaired by nonhomologous end joining (NHEJ), an efficient but error-prone mechanism that frequently causes small nucleotide insertions or deletions (indels) at 


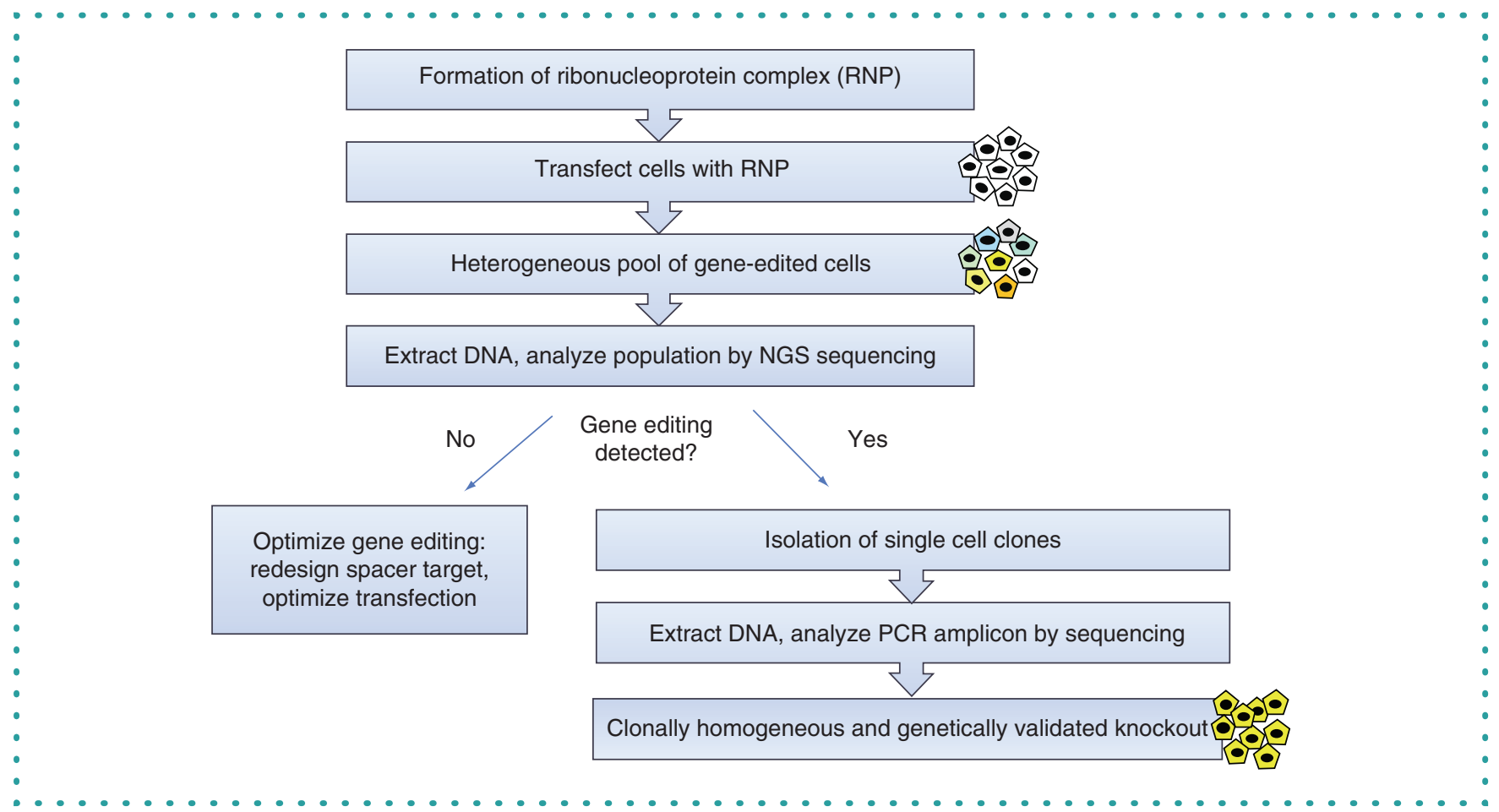

Figure 1. Schematic representation of the workflow to generate porcine gene knockout cells using CRISPR/Cas9. A ribonucleoprotein (RNP) complex is formed by incubating gRNA with Cas9. The RNP complex is transfected into cells using lipid nanoparticles resulting in a diverse array of mutations at the genomic target site in the cells. By isolation and sequence analysis of single cell clones, clonally homogenous cell lines with the desired edits can be obtained.

NGS: Next-generation sequencing.

the break site. These indels often result in amino acid deletions, insertions or frameshift mutations that create premature stop codons within the open reading frame of the targeted gene. Due to the randomness of NHEJ-mediated repair, a population of cells expressing Cas endonuclease and a gRNA will have a diverse array of mutations in the genomic target site [8].

Here we describe a streamlined approach for developing genetically tailored porcine HCC cells that can be used to develop precision models of HCC (Figure 1). As an example, we describe CRISPR/Cas9-mediated disruption of ARID1A in Oncopig HCC cells. ARID1A is a subunit of the SWI/SNF chromatin remodeling complex. It is mutated in several types of cancer, including HCC, and has a tumor suppressor role [10,11]. Most ARID1A mutations in tumors are inactivating frameshift or nonsense mutations that exist throughout the gene and result in loss of protein expression [10]. We introduce similar loss-of-function mutations in porcine ARID1A through Cas9mediated NHEJ and isolate homogenous cell clones with ARID1A knockout.

\section{Materials \& methods}

\section{Animal subjects}

Transgenic pigs (Sus scrofa) harboring Cre recombinase-inducible heterozygous $K R A S^{G 12 D}$ and $T P 53^{R 167 H}$ transgenes (Oncopigs) were used in this study [7]. All animal procedures were approved by the Institutional Animal Care and Use Committee at the University of Illinois at Chicago.

\section{Isolation of porcine hepatocytes}

Primary hepatocytes were isolated from the livers of Oncopigs $(n=5)$ as previously described [4]. A section of a liver lobe $(10-15 \mathrm{~g})$ was resected from each anesthetized Oncopig via a ventral midline laparotomy (Figure 2A \& B) and placed in ice-cold Krebs-Ringer buffer. The liver sample was flushed by cannulation into visible blood vessels on the cut surface using a catheter (\#8890703005; Covidien) (Figure 2C) with $250 \mathrm{ml}$ of buffer $\mathrm{A}(8.3 \mathrm{~g} / \mathrm{I} \mathrm{NaCl}, 0.5 \mathrm{~g} / \mathrm{l} \mathrm{KCl}, 2.4 \mathrm{~g} / \mathrm{l} \mathrm{HEPES}$ and $0.19 \mathrm{~g} / \mathrm{l} \mathrm{EGTA}$ at pH 7.4), followed by perfusion with $250 \mathrm{ml}$ buffer $\mathrm{B}(8.3 \mathrm{~g} / \mathrm{l} \mathrm{NaCl}, 0.5 \mathrm{~g} / \mathrm{l} \mathrm{KCl}$ and $2.4 \mathrm{~g} / \mathrm{l} \mathrm{HEPES}, \mathrm{pH}$ 7.4). Tissues were further perfused with $100 \mathrm{ml}$ digestion Buffer $\mathrm{C}$ (freshly prepared 0.015\% type IV Collagenase [\#17104019; Gibco/Thermo Fisher Scientific, MA, USA] in collagenase diluent [3.9 g/l NaCl, $0.5 \mathrm{~g} / \mathrm{I}$ $\mathrm{KCl}, 2.4 \mathrm{~g} / \mathrm{l} \mathrm{HEPES}, 0.7 \mathrm{~g} / \mathrm{I} \mathrm{CaCl}_{2} \cdot 2 \mathrm{H}_{2} \mathrm{O}$ at pH 7.4]). Next, the liver sections were perfused with $30 \mathrm{ml}$ buffer $\mathrm{D}$ (freshly prepared $0.15 \%$ collagenase type IV in collagenase diluent). The same buffer was used to perfuse the liver section 30-times until the liver became pale and the buffer became turbid with liberated cells. Buffers $A, B, C$ and D were maintained at $37^{\circ} \mathrm{C}$ throughout the procedure. Nonperfused 

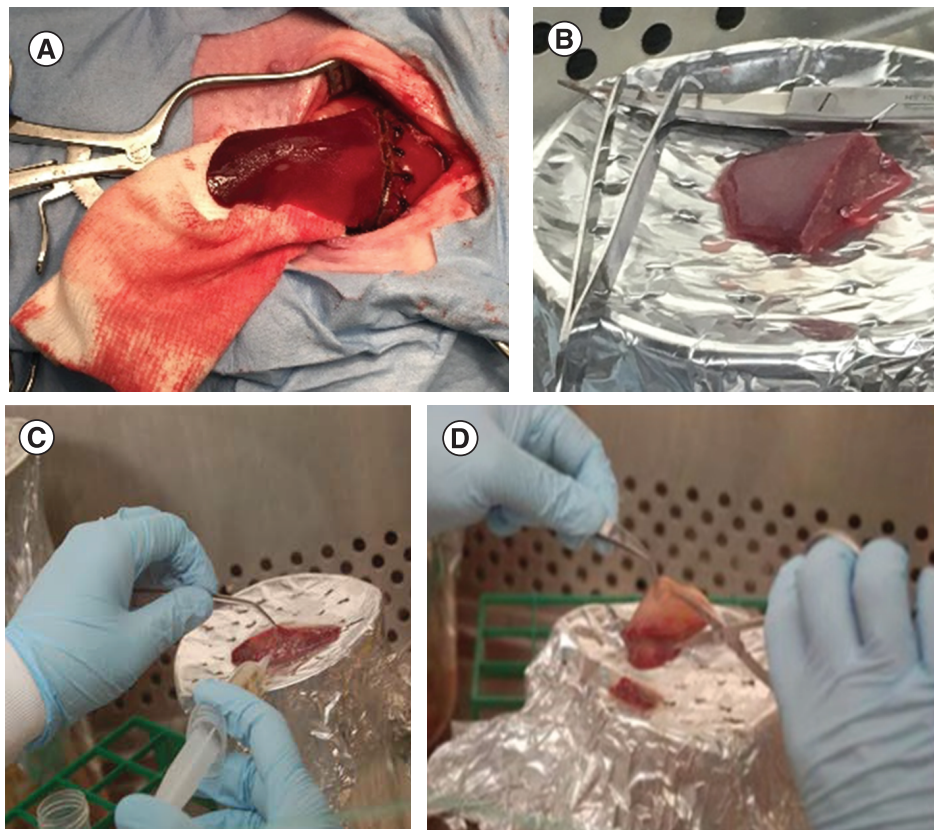

Figure 2. Isolation of hepatocytes from porcine liver section. (A \& B) A section of porcine liver is exposed and resected. (C) Cannulation of visible blood vessels on the cut surface of the liver section. (D) The liver section becomes pale after perfusion. Nonperfused red regions of the liver are discarded.

red regions of the liver were cut with a scalpel and discarded (Figure 2D). The remaining liver section was washed with $15 \mathrm{ml}$ ice-cold buffer E ( $9.91 \mathrm{~g} / \mathrm{l}$ Hanks' balanced salt solution without calcium and magnesium, $2.4 \mathrm{~g} / \mathrm{l} \mathrm{HEPES,} 0.35 \mathrm{~g} / \mathrm{l}$ sodium bicarbonate and $2 \mathrm{~g} / \mathrm{I}$ bovine serum albumin at $\mathrm{pH}$ 7.4). The resulting cell suspension (buffers $\mathrm{D}$ and $\mathrm{E}$ ) was filtered through a 100- $\mu \mathrm{m}$ nylon mesh (\#352360; BD Falcon) and centrifuged at $50 \times g$ for $4 \mathrm{~min}$ at $4{ }^{\circ} \mathrm{C}$. The pellet was re-suspended in $40 \mathrm{ml}$ ice-cold buffer 5 and filtered through a 70 - $\mu \mathrm{m}$ nylon mesh (\#352350; BD Falcon) and centrifuged at $50 \times g$ for $4 \mathrm{~min}$ at $4{ }^{\circ} \mathrm{C}$. The cell pellet was washed with $30 \mathrm{ml}$ ice-cold buffer $\mathrm{E}$ and centrifuged at $50 \times g$ for 6 min at $4{ }^{\circ} \mathrm{C}$, then the wash step was repeated two more times. After the last wash, cells were resuspended in Dulbecco's modified Eagle medium (DMEM) supplemented with 10\% fetal bovine serum (FBS) and 1\% penicillin-streptomycin.

\section{Generation of porcine HCC cells}

Isolated porcine hepatocytes were seeded into six-well plates at a density of $2.5 \times 10^{6}$ cells/well. The next day, the medium was replaced with low-serum DMEM (5\% FBS). Adenoviral vector with cytomegalovirus promoter driving the expression of Cre recombinase fused to enhanced green fluorescent protein (Ad5CMVCre-eGFP; University of lowa Vector Core, IA, USA) was added at a 400 multiplicity of infection. The medium was changed to DMEM containing 10\% FBS at $4 \mathrm{~h}$ post-transduction. Expression of the transgene and hepatocellular origin of the cells were confirmed by RT-PCR and arginase-1 staining, respectively, as previously described $[4,5]$.

\section{Cell culture}

Porcine HCC cells were maintained in DMEM supplemented with $10 \%$ FBS and $1 \%$ penicillin-streptomycin. All culture media and supplements were purchased from Gibco/Thermo Fisher Scientific.

\section{gRNA design}

CRISPOR design software (www.crispor.tefor.net) was used to predict the optimal genomic target for ARID1A gene knockout [12]. The software design parameters were set to the $S$. scrofa 11.1 reference genome and the 20 -nucleotide spacer with a PAM sequence of NGG specific for the Streptococcus pyogenes Cas9 nuclease. Two sites with minimal off-target sequence similarity in the $S$. scrofa genome were selected and tested (Table 1). The sites were located in an early protein-coding sequence in ARID1A to ensure target ablation.

\section{Preparation of gRNAs}

The AltR ${ }^{\circledR}$ CRISPR gene editing system (IDT Corporation, IL, USA) was used for editing porcine HCC cells. Each gRNA was synthesized by incubating equimolar ratios of crRNA (sequences in Table 1, synthesized by IDT Corporation) and tracrRNA labeled at the $5^{\prime}$ end with ATTO 550 fluorescent dye (\#1075927; IDT Corporation) at $95^{\circ} \mathrm{C}$ for $5 \mathrm{~min}$, followed by cooling to room temperature. 


\section{Table 1. Oligonucleotides used in the study. \\ crRNAs \\ ARID1A CrRNA\#1 5'-GGACTTTGCTGGTTGTAATA-3' \\ ARID1A CrRNA\#2 5'-CAACAAGGCCCCAGCGGGTA-3' \\ PCR primers for targeted sequencing \\ ARID1A-For 5'-ACACTCACGACATGGTTCTACATAAACTACCAGAAGTATCAGTGCT-3' \\ ARID1A-Rev 5'-TACGgTAGCAGAGACTTGGTCTTGGCTGCTGGGAATATGGAG-3'}

The bold regions represent adaptor sequences for barcode attachment.

\section{Transfection of RNP complexes}

Purified S. pyogenes Cas9 nuclease (\#1081058; IDT Corporation) was diluted in Opti-MEM (\#31985062; Gibco/Thermo Fisher Scientific), then combined with gRNA at equimolar ratio to form a RNP complex. Porcine HCC cells were reverse transfected with $15 \mathrm{nM}$ or $25 \mathrm{nM}$ RNP using Lipofectamine CRISPRMAX transfection reagent (\#CMAX00003; Invitrogen, CA, USA) following the manufacturer's instructions.

\section{Analysis of gene editing by next-generation sequencing}

Genomic DNA was extracted from porcine HCC cells using QuickExtract DNA Extraction Solution (\#QE09050; Lucigen, WI, USA) as follows: cells were washed with phosphate-buffered saline (PBS) then collected in QuickExtract solution and heated in a thermal cycler at $65^{\circ} \mathrm{C}$ for $10 \mathrm{~min}$, followed by $98^{\circ} \mathrm{C}$ for $5 \mathrm{~min}$. The genomic locus that flanks the Cas 9 target site was amplified by PCR using the primers listed in Table 1. A second PCR was done to attach Fluidigm barcode sequences to the amplicons generated. Targeted next-generation amplicon sequencing (NGS) was performed using a MiSeq instrument $(2 \times 150$ kit, Illumina, CA, USA) following the manufacturer's instructions. The gene editing efficiency was calculated using the CRISPRESSO2 alignment tool (www.crispresso.pinellolab.partners.o $\mathrm{rg} /$ submission) [13]. This program aligns the raw FASTQ files generated by the Illumina miniSeq to a user-supplied reference sequence. The algorithm has improved upon the Needleman-Wunsch alignment to consider guide sequence, predicted cleavage site and nuclease properties. The inclusion of the gene editing biology in the NGS analysis increases the accuracy of indel calling compared with basic analysis methods. The standard program parameters were applied to calculate the gene editing in the three-base window around the predicted cleavage site and the gene editing efficiency was determined by quantifying the frequency of sequences containing indels.

\section{Analysis of gene editing by Sanger sequencing}

Amplification of genomic DNA using the primers listed in Table 1 was performed as described above. The amplicons were analyzed by Sanger sequencing using the ARID1A-Rev primer. The sequencing files were uploaded and analyzed by the online software Inference of CRISPR Edits (ICE; ice.synthego.com).

\section{FACS}

At 2 days post-RNP transfection, the cells were washed twice with PBS, detached with trypsin and resuspended in culture medium. FACS was performed using a MoFlo Astrios cell sorter (Beckman Coulter, CA, USA). The sorted cells were collected in an Eppendorf tube for DNA extraction or seeded as single cells into two 96 -well plates for growing single-cell clones.

\section{Isolation of single cell clones}

For cells not sorted by FACS, the cell suspension was diluted to 1 cell per $100 \mu \mathrm{l}$ medium and single cells were seeded into each well of a 96-well plate. Visual inspection was done to confirm the presence of a single cell/colony per well. When a colony reached about $80 \%$ confluency, cells were trypsinized and reseeded into two wells of another 96 -well plate. When cells reached confluency, one well was used for DNA extraction using $20 \mu \mathrm{l}$ QuickExtract DNA Extraction solution and analyzed by NGS as described above; the other well was expanded by seeding into a 24-well plate, then cryopreserved.

\section{Immunofluorescence staining}

Cells were seeded in glass chambers (\#PEZGS0416, Millipore Sigma) and kept in a cell culture incubator at $37^{\circ} \mathrm{C}$ overnight. Next day, the cells were washed with PBS and fixed with ice-cold methanol for $5 \mathrm{~min}$. Then the cells were washed and permeabilized with PBS containing $0.1 \%$ Triton $^{\top M} \mathrm{X}-100$ for $10 \mathrm{~min}$, washed again and blocked for $30 \mathrm{~min}$ with $1 \%$ bovine serum albumin in a solution of PBS and $0.1 \%$ Tween 20 . Next, cells were incubated with anti-arginase primary antibody (\#ab91379; Abcam) overnight at $4^{\circ} \mathrm{C}$, followed by washing and $1 \mathrm{~h}$ incubation with secondary antibody conjugated to Alexa Fluor ${ }^{\circledR} 488$ (\#A27034; Invitrogen/Thermo Fisher Scientific) at room temperature. The cells were washed again and nuclei were stained with $0.1 \mu \mathrm{g} / \mathrm{ml}$ DAPI (\#40043; Biotium) before being washed and visualized using a fluorescence microscope. 


\section{Western blotting}

$2.5 \times 10^{6}$ cells were seeded in six-well plates and kept in a $37^{\circ} \mathrm{C}$ cell culture incubator overnight. Next day, the cells were washed with ice-cold PBS and lysed with $250 \mu$ l RIPA buffer (\#AAJ63306AK, Thermo Fisher Scientific) mixed with Halt Protease Inhibitor Cocktail (\#78429, Thermo Fisher Scientific). SDS-PAGE was performed, then the proteins were transferred onto nitrocellulose membranes and the membranes blocked with $5 \%$ nonfat milk in PBS with Tween 20 for $30 \mathrm{~min}$. Incubation with the primary antibodies was done overnight at $4{ }^{\circ} \mathrm{C}$ followed by $1 \mathrm{~h}$ incubation with the appropriate secondary antibodies at room temperature. The following anti-ARID1A primary antibodies were used: \#sc-373784 and \#sc-32761 (Santa Cruz Biotechnolog, TX, USA) and \#A301-041A (Bethyl Laboratories, TX, USA). Anti-mouse and anti-rabbit secondary antibodies were used (\#170-6516 and \#170-6515, Bio-Rad, CA, USA). The western blots were visualized by chemiluminescence (\# 32109, Thermo Fisher Scientific).

\section{Results \& discussion}

Porcine ARID1A gene targeting

To investigate the contribution of clinically relevant mutations on malignant potential and treatment susceptibility using a large animal model, we aimed to target ARID1A in porcine HCC cells. First, we assessed the similarity between the human and porcine ARID1A genes to establish the clinical relevance. This comparison revealed a high degree of homology, wherein human and porcine ARID1A gene sequences shared $89 \%$ identity and the coding regions revealed $95 \%$ identity. To generate loss-of-function mutations in ARID1A similar to those occurring in human cancers, we designed two individual ARID1A-targeting gRNAs (Figure 3A). Testing more than one gRNA is important for validation of in silico predictions of efficiency, especially because factors other than DNA sequence may affect gRNA efficiency at a given site [14]. It is important to target early in the gene coding region so that indels formed in the beginning of the coding sequence can lead to an out-of-frame shift, which frequently results in an early stop codon, nonsense-mediated decay of the mRNA and eventually no production of the protein.

One of the major factors that affects gene editing efficiency in a cell population is the successful delivery of CRISPR components to cells. The gRNA and Cas 9 can be introduced into cells as DNA, RNA, or precomplexed RNPs [15]. Delivery of RNPs has several advantages compared with gene delivery: reduced off-target effects, transient presence of Cas9 in cells and increased editing efficiency [15-17]. Hence we assembled RNP complexes and transfected them into cells.

Porcine HCC cells treated with $15 \mathrm{nM}$ Cas 9 and ARID1A-targeting gRNA\#1 or gRNA\#2 were successfully edited at the expected target region, as demonstrated by NGS. The gene editing efficiencies in cells transfected with gRNA\#1 or gRNA\#2 were 52.7 and $10.8 \%$, respectively (Figure 3B). As gRNA\#1 resulted in a higher editing efficiency than gRNA\#2, it was used for all further experiments. Consistent with the anticipated CRISPR/Cas 9 effect, small indels occurred around the predicted Cas 9 cleavage position (Figure 3C \& D). As expected, nontransfected control cells did not show any NHEJ events at the target site (Figure 3B). These results demonstrate successful CRISPR/Cas9 disruption of the porcine ARID1A gene.

\section{Increasing the yield of gene-edited cells}

Though transfection of precomplexed RNPs has several advantages compared with DNA delivery methods, it precludes the ability to select transfected cells based on features such as antibiotic resistance. As an alternative, we used a fluorescently labeled tracrRNA to form the RNP and isolated transfected cells by FACS (Figure 4A). This approach allows visual assessment of transfection efficiency, as the intracellular uptake of the gRNA can be detected using a fluorescence microscope. Indeed, FACS-sorted porcine HCC cells transfected with $15 \mathrm{nM}$ RNP comprising Cas9 and gRNA\#1 targeting ARID1A had 79\% editing efficiency, as compared with 65\% editing in unsorted cells (Figure 4B). This demonstrates the feasibility of increasing the yield of edited cells by enriching the transfected cell fraction.

Next, we aimed to increase the transfection efficiency by increasing the concentration of transfected RNPs to avoid the need for FACS. By increasing the RNP concentration to $25 \mathrm{nM}$ with an appropriate increase of the transfection reagent, ARID1A editing in four porcine HCC cells was greater than $89 \%$ (Figure 5). Transfection with RNP concentrations higher than $25 \mathrm{nM}$ was not feasible due to the remarkable rate of cell death, likely caused by the increased amount of transfection reagent. This highlights the importance of optimizing transfection conditions in different cell types to achieve the highest possible transfection efficiency without compromising cell viability.

Notably, the identities and frequencies of indels were similar in all the cell lines treated with the same gRNA (Figure 5B), consistent with previous studies [14,18]. Interestingly, the highest-frequency indel across all the cell lines was a T nucleotide insertion. This is in line with a recent study which demonstrated that single-nucleotide indels are the most frequent type of indel for the majority of gRNAs, and that target sites that contain A or T upstream of the cleavage site are most frequently repaired by insertion of A or T, respectively [14].

\section{Analysis of CRISPR/Cas9-mediated gene disruption}

NGS is considered the gold standard for analyzing CRISPR editing outcomes because of its high accuracy and sensitivity. However, NGS is also time-consuming and expensive and hence not ideal for preliminary screening experiments. We compared the analysis of Cas9mediated ARID1A edits in an Oncopig HCC cell line by NGS and Sanger sequencing. Due to the expected heterogeneity of modifications at the gRNA target site, Sanger sequencing of the PCR amplicons produced from a pool of cells superimposed sequencing chromatograms and displayed overlapping peaks near the expected Cas9 cleavage site (Figure 6A). Several software tools were developed recently to 
(A)

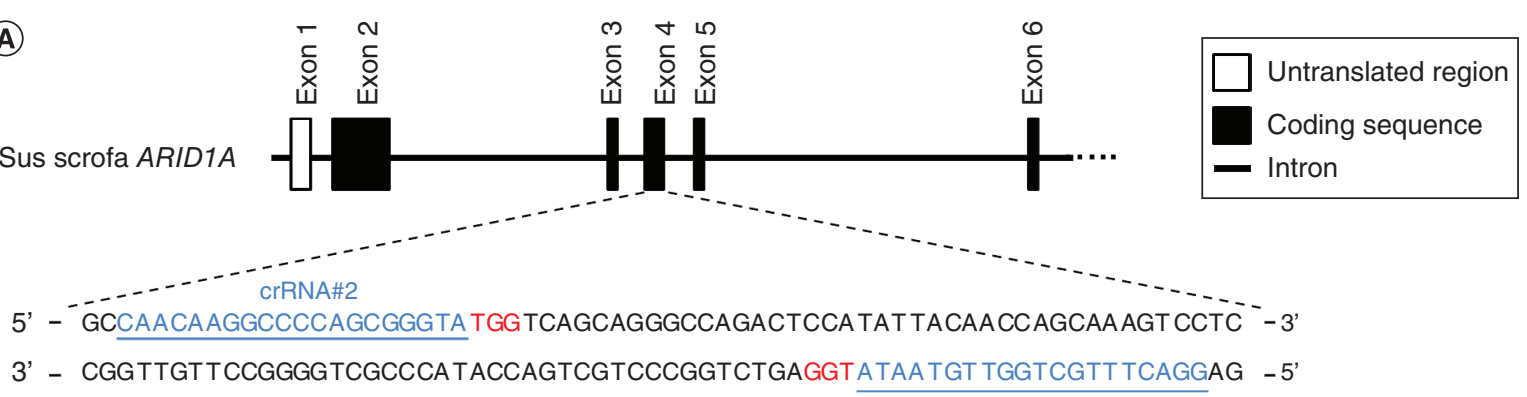

CrRNA\#1

(B)

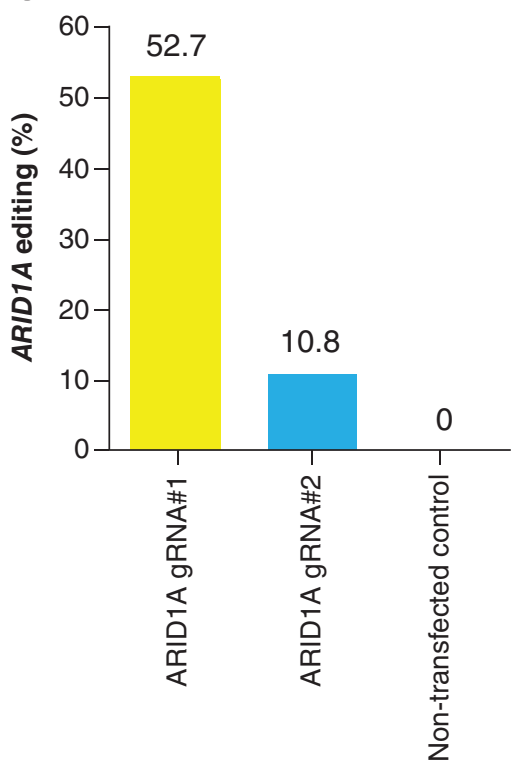

(D)

\begin{tabular}{|cl|}
\hline Bold & Substitutions \\
$\square$ & Insertions \\
- & Deletions \\
----- & Predicted cleavage position \\
\hline
\end{tabular}

(C)

\section{Cas9 mediated disruption of ARID1A by gRNA\#1}

\section{A GCAGGGC CAGACTCCATATTACA A C CAGCA A}

A GCA G G C CA A C TC CA TA TITA CAACCAGCAA G CAGGGCCAGACTCCA T ATTTT A C A A C C A G C A A A GCAGGGC CA A C TC C A - ITA C A A C CAGCAA A GCAGGGCCAGACTCCA TA T A CA A C CAGCA A A GCAGGGC CAGACT ....... C CAGCAA A GCAGGGCCAGACTCC - A TT T C A A C CAGCA A A GCAGGGC CAG ......... A C A A C C AGCA A A GCA G GGC CAGACTCCATATATACA ACCAGCA A GCAGGGC CAGACTC - ... - CAACCAGCAA A GCAGGG ......... C CAGCAA A GCAGGGC CAGACTCCATAT- CAACCAGCAA AGCAGGGC CAGACTCCA TA T . A A C CAGCAA A GCA A G C C CA GC C TC C A T A TITA CA A C CAGCAA A GCAGGGC CAGACTCCA ... GACAACCAGCAA

Reference

$36.3 \%$ * $11.7 \%$ $6 \%$ $5.3 \%$ $3.3 \%$ $2.2 \%$ $1.5 \%$ $1.1 \%$ $1.1 \%$ $1.1 \%$ $1.0 \%$ $0.8 \%$ $0.7 \%$ $0.6 \%$ $0.5 \%$

\section{Case 9 mediated disruption of ARID1A by gRNA\#1}

GC C C $\triangle A G C G G G T A T G G T C A G C A G G G C \subset A G A$ Reference

GC C C C A G C G G T A T G G T A G C A G G C C A A $74.5 \%$ * C C C C A G CGGGG A T G GTCA G C A G G C C A G A $2.4 \%$ GC C C CA GC. GI TATGGTCAGCA GGGC C A G A $2 \%$ GCC C C A GCGGG T T G G T C A G CA GGGC C A GC $1.6 \%$ G C C C C A GC - . - G G T C A G C A G G G C C A G A $1.0 \%$ GC C C . . . . . . C A G C A GGGC C A G A $0.9 \%$ GCC C CA .... T T T G GT C A GCA GGGCCA G A $0.7 \%$ G C C C C A GC - G T A T G G T C A G C A G G G C C A G A $0.6 \%$ GC C C C A GCG G C T A T G G T C A G C A G G G C C A G $0.5 \%$ GC $C \subset \subset A G C G G T$ T $T$ A T G G T C A GC A G G G C C A G G C C C C A GC G Gi- - A T G G T C A G C A G G G C C A G A $0.3 \%$ $G \subset \subset \subset C C G C G G G T A T G G T C A G C A G G G C \subset A G A$ $G C C C C A G C G G G T A T G G T C A G C A G G G C C A G A$ G C C C C A GC G- - - G G T C A G C A GG G C C A G A $0.3 \%$ GC C C CAGC - - . T G G T CAGCAGGGC C A G A $0.3 \%$

Figure 3. CRISPR/Cas9-mediated disruption of the porcine ARID1A gene. (A) Schematic representation of the porcine ARID1A locus, showing the location of spacer sequences crRNA\#1 and crRNA\#2 (underlined blue font). Protospacer-adjacent motif sequences are marked in red. The introns are represented by lines, coding regions of exons are shaded in black and the untranslated region is white. (B) Comparing the CRISPR/Cas9-mediated editing efficiency of two ARID1A-targeting gRNAs. Porcine HCC line-1 cells were transfected with 15 nM RNP comprising Cas9 and gRNA\#1 or gRNA\#2. Nontransfected cells were used as control. Genomic DNA was collected 2 days post-transfection and analyzed by targeted NGS. The bar graph depicts the percentages (\%) of total reads that displayed indels at the gRNA target site occurring as a result of nonhomologous end joining. (C \& D) Top 15 reads detected by targeted NGS analysis, mapped to the reference sequence. The percentages of reads for each sequence are shown on the right. The asterisk indicates non-edited reads. Dashed line: predicted Cas 9 cleavage position; red box: insertion; dash: deleted base.

HCC: Hepatocellular carcinoma; NGS: Next-generation sequencing; RNP: Ribonucleoprotein. 
(A)

Fluorescent tracrRNA + crRNA

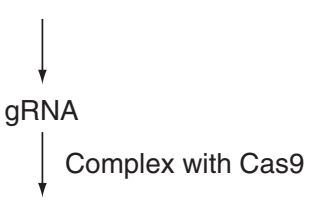

RNP<smiles>[3H][CH]</smiles>

Transfect into cells $48 \mathrm{~h}$

FACS to separate fluorescent cells
(B)

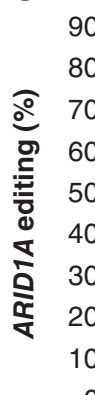

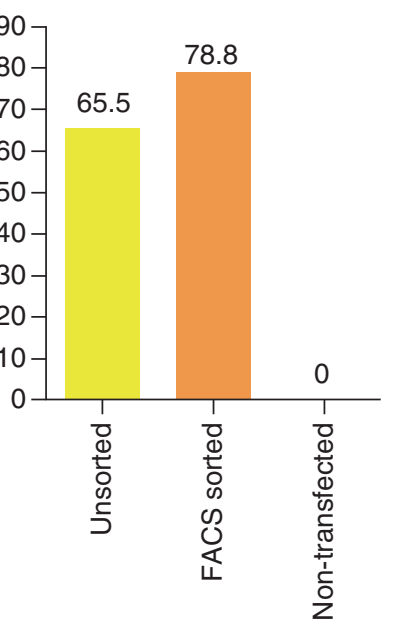

Figure 4. Fluorescence-activated cell sorting increases the percentage of gene-edited cells. (A) Schematic representation of the workflow to sort RNP-transfected cells. A tracrRNA conjugated with ATTO-550 fluorescent dye was mixed with crRNA to form gRNA. The fluorescently labeled gRNA and purified Cas 9 were complexed to form a ribonucleoprotein complex (RNP) which was then transfected into cells, allowing visual assessment of transfection efficiency using a fluorescence microscope. $48 \mathrm{~h}$ later, the cells were sorted by FACS to separate successfully transfected fluorescent cells. (B) ARID1A editing efficiency in unsorted or FACS-sorted porcine HCC cells. Porcine HCC line-1 cells were transfected with $15 \mathrm{nM}$ RNP comprising Cas9 and ARID1A targeting gRNA\#1. Nontransfected control cells were also analyzed. FACS-sorted cells were used for genomic analysis directly after sorting. The bar graph depicts the percentages (\%) of total reads that displayed indels at the gRNA target site as analyzed by NGS. HCC: Hepatocellular carcinoma; NGS: Next-generation sequencing.

interpret overlapping peaks arising from a mixture of indels and assess genome editing efficiency using Sanger sequencing data. We found that the editing efficiency predicted by Sanger sequencing data analysis was quite similar to that predicted by NGS $(89.3 \pm 2.5 \%$ vs $91.5 \pm 2.5 \%$ respectively) (Figure 6B). Also, the sequence and abundance of the most frequent indels was fairly similar (Figure 6B \& C). For example, both analyses identified a single nucleotide insertion to be the most frequent indel. However, many types of indels were not captured by Sanger sequencing data analysis. Overall, these results show that Sanger sequencing data analysis can provide a rough estimation of editing efficiency and identify some of the most frequent types of indels, whereas NGS is preferable when a more accurate and comprehensive analysis of CRISPR/Cas editing outcome is needed.

\section{Isolation of homogenous knockout clones}

Since CRISPR/Cas9 results in diverse modifications, including in-frame indels that do not cause functional gene knockout, it is frequently necessary to isolate and screen homogenous pools of cells. Our purpose is to develop genetically tailored HCC tumors in pigs to investigate the effects of gene knockout on HCC tumor progression and susceptibility to targeted therapies; hence it is important to achieve and validate gene knockout in the developed HCC cells before orthotopic implantation into pigs. We isolated single-cell clones from FACS-sorted or unsorted ARID1A-edited cells and analyzed gene edits by NGS. There are several possible outcomes for each isolated clone: absence of mutations in both alleles, heterozygous mutation (where one allele is edited and the other is not), homozygous mutation (where both alleles are edited identically) or biallelic mutation (in which the alleles have different edits) (Figure 7A). All these outcomes were represented in the 14 clones that we isolated and screened (Table 2 \& Figure 7B). Indels that affect bases in multiples of 3 are less likely to result in a functional knockout and hence were excluded from further downstream analysis. All other indels appearing in the clones caused frameshift mutations and premature stop codons and were predicted to result in production of a truncated ARID1A protein (Figure 7B \& C).

Next we confirmed the hepatocellular origin of each of the isolated single-cell clones. Our developed HCC cell lines typically comprise 88-100\% hepatocytes that are immunoreactive to arginase- 1 antibody staining [5]. This necessitates testing the cellular origin of the isolated clones to exclude nonhepatocyte clonal populations. All the isolated single-cell clones showed positive arginase-1 staining, confirming their hepatocyte origin (Figure 7D).

In addition to confirmation of edits on a genetic level, it is important to confirm target protein loss. We attempted to confirm ARID1A protein loss following CRISPR editing by performing western blotting analysis using several commercially available antibodies that target the human ARID1A protein. However, none of these antibodies were immunoreactive toward the porcine ARID1A protein, necessitating the testing of additional antibodies for cross-reactivity or the generation of a custom antibody specific for porcine ARID1A. The lack of commercially available porcine antibodies represents a major challenge when using pigs as disease models, because most commercially 
(A)

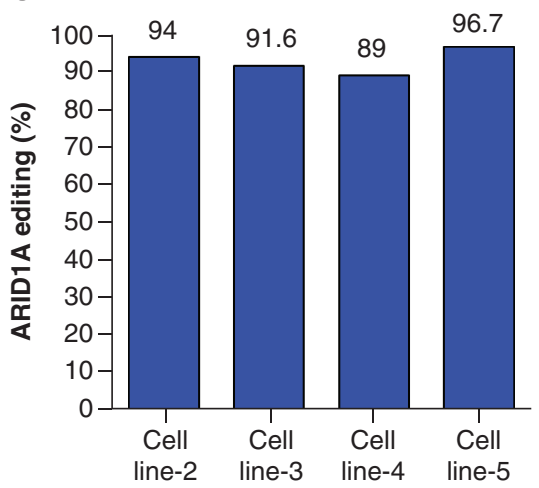

(B)

NGS analysis of ARID1A in porcine HCC cell lines

A G G G C A G A C T C C A T A T T A C A A C A G C A A A G T R Reference

1:1i A G G G C C A G A C T C C A T A T TTT A C A A C C A G C A A A G T $24.1 \%$

1:1d A G G G C C A G A C T C C A T A T A A C A A C C A G C A A A G T C $14.1 \%$

-1:3d A G G G C C A G A C T C C A T A - C A A C C A G C A A A G T C $6.7 \%$ No variant A G G G C C A G A C T C C A T A T T A A C A A C C A G C A A A G T C $5.4 \%$ *

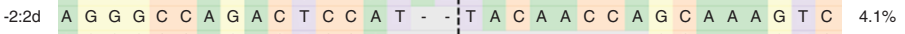

-3:11d A G G G C C A G A C T C C A . . . . . . . G C A A A G T C $4.1 \%$

1:1i A G G G C C A G A C T C C A T A T A T A C A A C C A G C A A A G T $3.0 \%$

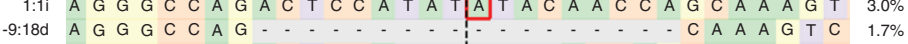

3:7d A G G G C C A G A C T C C A

1:2d A G G G C C A G A C T C C A T A T - A C C A G C A A A G T C $1.7 \%$

C A T A T:- - C A A C C A G C A A A G T C $1.7 \%$

7:10d A G G G C C A G A C - ....... A A C C A G C A A A G T C $1.6 \%$

1:3d A G G G C C A G A C T C C A T A T - - A A C C A G C A A A G T C $1.1 \%$

1:4d A G G G C C A G A C T C C A T A T - . A C C C A G C A A A G T C $0.9 \%$

16.42 $A$ -

$-16: 27 \mathrm{~d}$ A \begin{tabular}{llllll} 
A & $A$ & $G$ & $T$ & $C$ & $0.9 \%$ \\
\hline
\end{tabular}

\section{Cell line-2}

1:1i A G G G C C A G A C T C C A T A T TT T A C A A C C A G C A A A G T $30.7 \%$ 1:1d A G G G C C A G A C T C C A T A T:A C A A C C A G C A A A G T C $10.0 \%$ Novariant A G G G C C A G A C T C C A T A T T A C A A C C A G C A A A G T C

$-1: 3 d$ A G G G C C A G A C T C C A T A - C C A A C C A G C A A A G T C $4.5 \%$

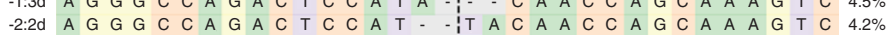

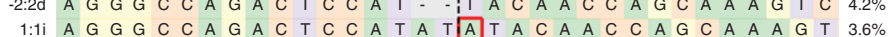

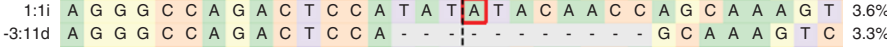

1:2d A G G G C C A G A C T C C A T A T - C A A C C A G C A A A G T C $2.0 \%$

$-9: 18 d$ A G G G C C A G

$-7: 10 d$ A G G G C C A G A C C A A A G T C $1.6 \%$

- A A C C A G C A A A G T C $1.2 \%$

3.7d A G G G C C A G A C T C C A - . - . A C C A G C A A A G T C $0.9 \%$

1:3d A G G G C C A GACTCCA T A T - A A C C A GCA A A G T C $0.8 \%$ $16: 27 \mathrm{~d}$ A $\ldots \ldots \ldots$. . . . . . . . A A G T C $0.6 \%$

1:4d A G G G C C A G A C T C C A T A T - . A C C A G C A A A G T C $0.6 \%$

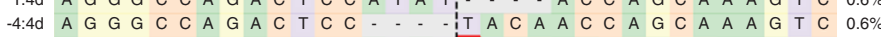

\section{Cell line-3}

1:1i A G G G C C A G A C T C C A T A TTT T A C A A C C A G C A A A G T $28.0 \%$ 1:1d A G G G C C A G A C T C C A T A T - A C A A C C A G C A A A G T C $9.1 \%$ No variant A G G G C C A G A C T C C A T A TIT A C A A C C A G C A A A G T C $8.3 \%$

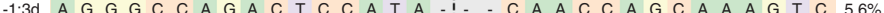

1:1i A G G G C C A G A C T C C A T A T A T A C A A C C A G C A A A G T $3.2 \%$

$-9: 18 d$ A G G G C C A G

-7:10d A G G G C C A G A

-3:7d A G G G C C A G A C T C C A -

$1.4 d A G G G C C A G A C T C C A T A T$

$16: 27 \mathrm{~d}$ A $\ldots \ldots \ldots$ A

C A A C C A G C A A A G T C $1.8 \%$

A A C C A G C A A A G T C $1.7 \%$

A C C A G C A A A G T C $1.5 \%$

A C C A G C A A A G T C $0.7 \%$

1:1i A G G G C C A G A C T C C A T A T G T A C A A C C A G C A A A G T $0.5 \%$

$-7: 13 d$ A $G$ G G C C A G A C C A G C A A A A T C $0.5 \%$

\section{Cell line-4}

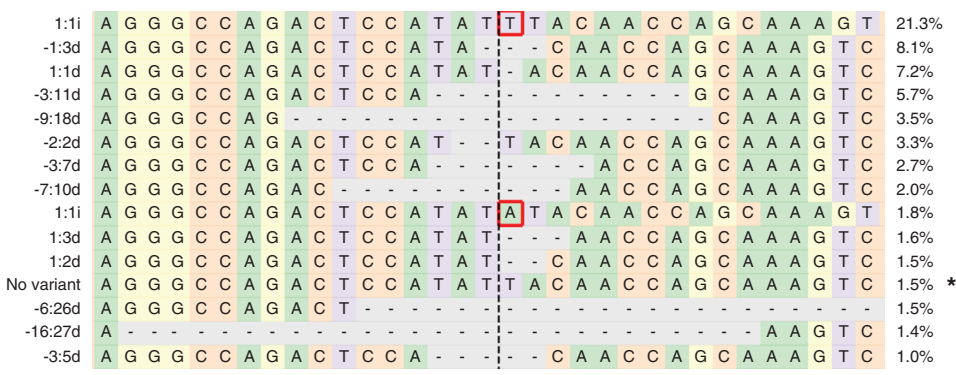

Cell line-5

\begin{tabular}{|cl|}
\hline$\square$ & Insertions \\
- & Deletions \\
----- & Predicted cleavage position
\end{tabular}

Figure 5. Increased CRISPR-Cas9 editing efficiency of ARID1A at higher ribonucleoprotein concentration. (A) ARID1A editing efficiency in four porcine $\mathrm{HCC}$ cell lines following transfection with $25 \mathrm{nM}$ ribonucleoprotein comprising Cas 9 and gRNA\#1. Genomic DNA was collected 2 days post-transfection and analyzed by targeted NGS. The bar graph depicts the percentages (\%) of total reads that displayed indels at the gRNA target site occurring as a result of nonhomologous end joining. (B) Top 15 reads detected by targeted NGS analysis in the four porcine HCC lines, mapped to the reference sequence. The identity and frequency of indels in the four cell lines is consistent. The percentages of reads of each sequence are shown on the right. The location of the indel relative to the expected cleavage site is shown on the left, followed by a colon and the number of nucleotides inserted (i) or deleted (d). The asterisk denotes non-edited reads. Dashed line: predicted Cas9 cleavage position; red box: insertion; dash: deleted base. HCC: Hepatocellular carcinoma; NGS: Next-generation sequencing. 
(A) ARID1A gRNA treated

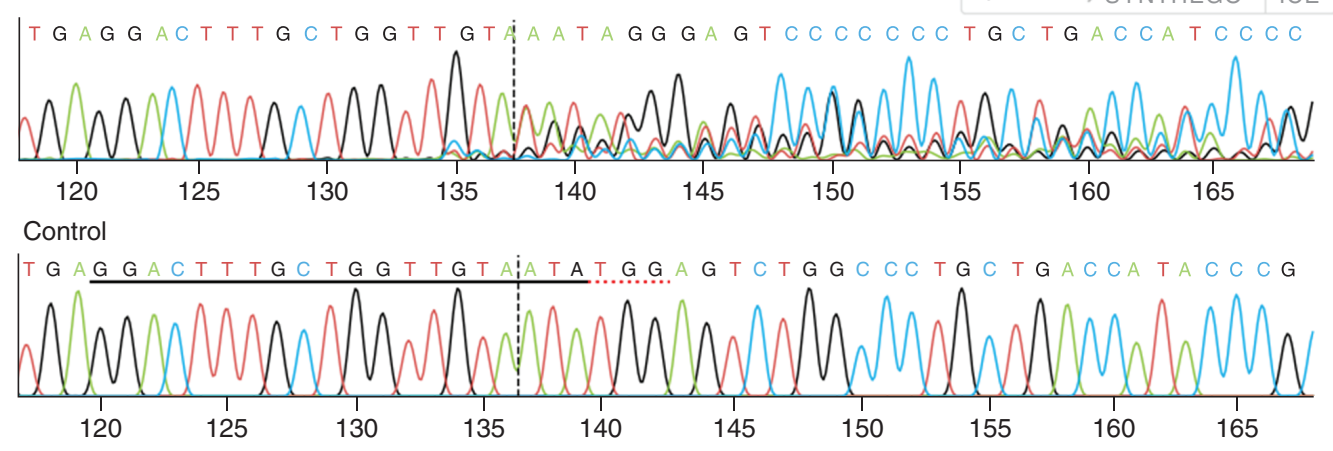

(B)

Sanger sequencing analysis of ARID1A in porcine HCC cell lines

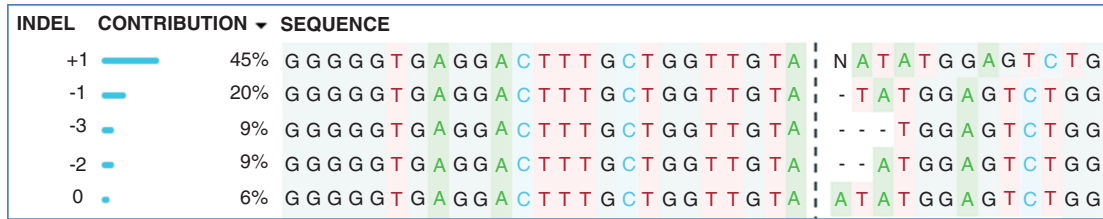

INDEL CONTRIBUTION 、 SEQUENCE

$+1=57 \%$ GGGGGGAGGCTT TGCTGGT TGTA N A TATGGAGT T T

$1-14 \%$ GGGGTGAGGCTTTGCTGGTTGTA: - TATGGGTCTGG

$-2 \quad 10 \%$ GGGGGGAGGACTTGCTGGTTGTA $\quad$ - A TGGAGTCTGG

-3 - $\quad 7 \%$ GGGGGGAGGATTTGCTGGTTGTA: - . TGGAGTCTGG

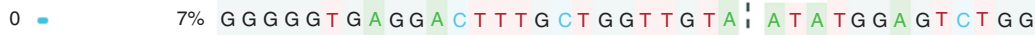

Cell line-3

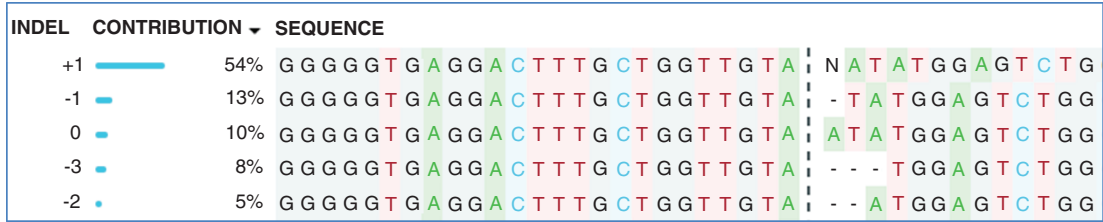

Cell line-2

(C)

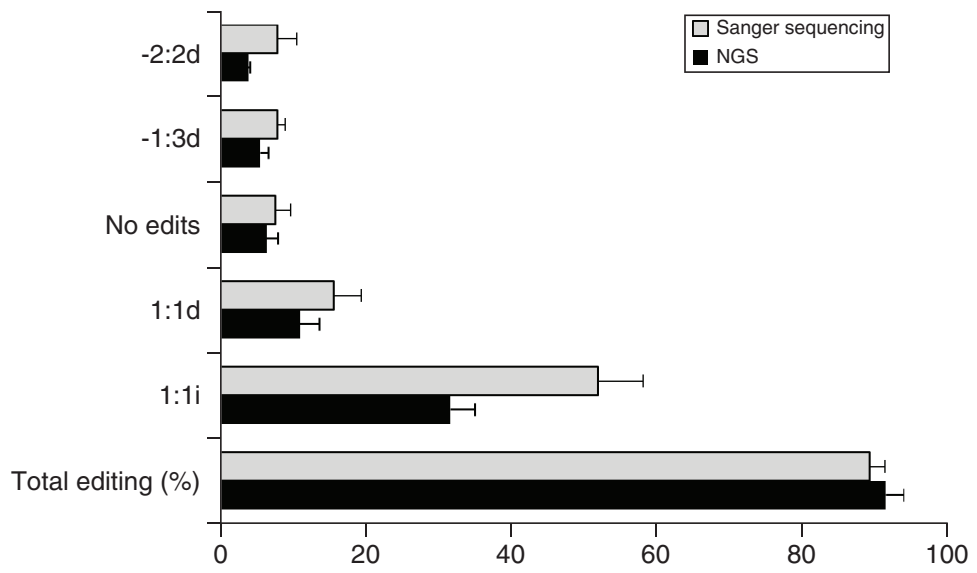

Figure 6. Comparing analysis of CRISPR/Cas9 mediated disruption of ARID1A by next-generation and Sanger sequencing. (A) Sanger sequencing chromatograms of porcine HCC cells transfected with Cas9 and ARID1A gRNA\#1 and control nontransfected cells. Overlapping peaks near the gRNA target site are observed for the sample subjected to CRISPR/Cas9 editing. Dashed line: predicted Cas9 cleavage position; underlined nucleotides: crRNA spacer sequence. (B) Top 5 reads detected by ICE software analysis of Sanger sequencing data in three porcine HCC lines transfected with Cas 9 and ARID1A gRNA\#1. The type and frequency of indels is shown on the left and is consistent among the three cell lines. (C) The editing efficiency (\%) and frequency of the top five frequent reads (\%) identified by ICE software analysis of Sanger sequencing data as compared with NGS analysis in three ARID1A-edited porcine HCC cells. The location of the indel relative to the expected cleavage site is shown on the left, followed by a colon and the number of nucleotides inserted (i) or deleted (d). Overall similarity was noted between the two analysis methods. HCC: Hepatocellular carcinoma; NGS: Next-generation sequencing. 
(A)

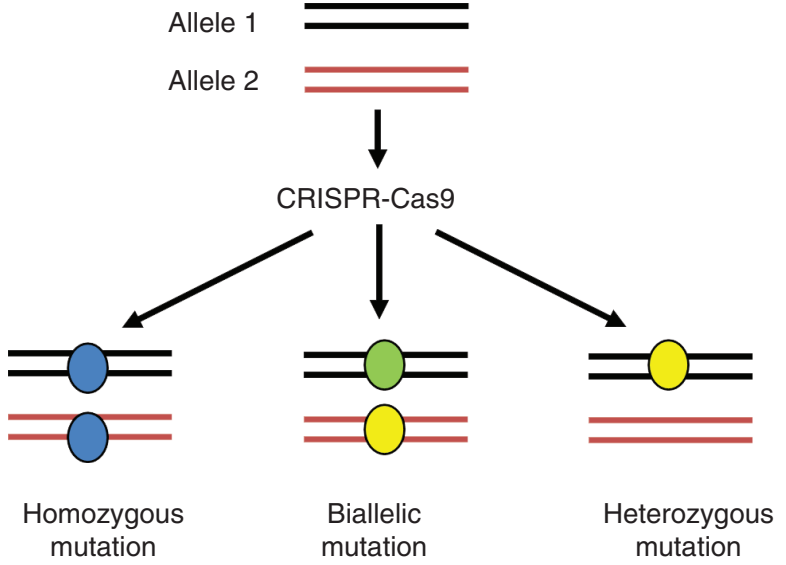

(B)

Reference C A G A C T C C A T A T T A C A A C C A G

Clone 1 C A G A

C A G A C T C C A T A - - - - A C C A G

Clone 2 C A G A C T C C A - . - - A C C A G

Clone 3

C A G A C - - - - - - A A C C A G (-10) C A G A $C$ C T C C C A T A T T

(C)

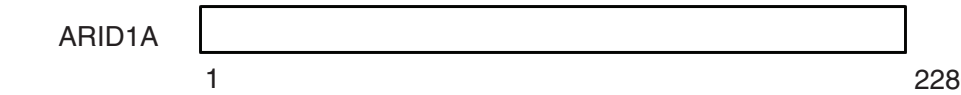

Clone $1(+1)$
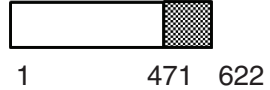

Clone $2(-5)$

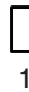

Clone $2(-7)$

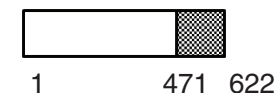

Clone $3(-10)$

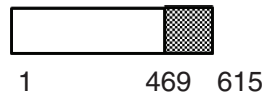

(D)

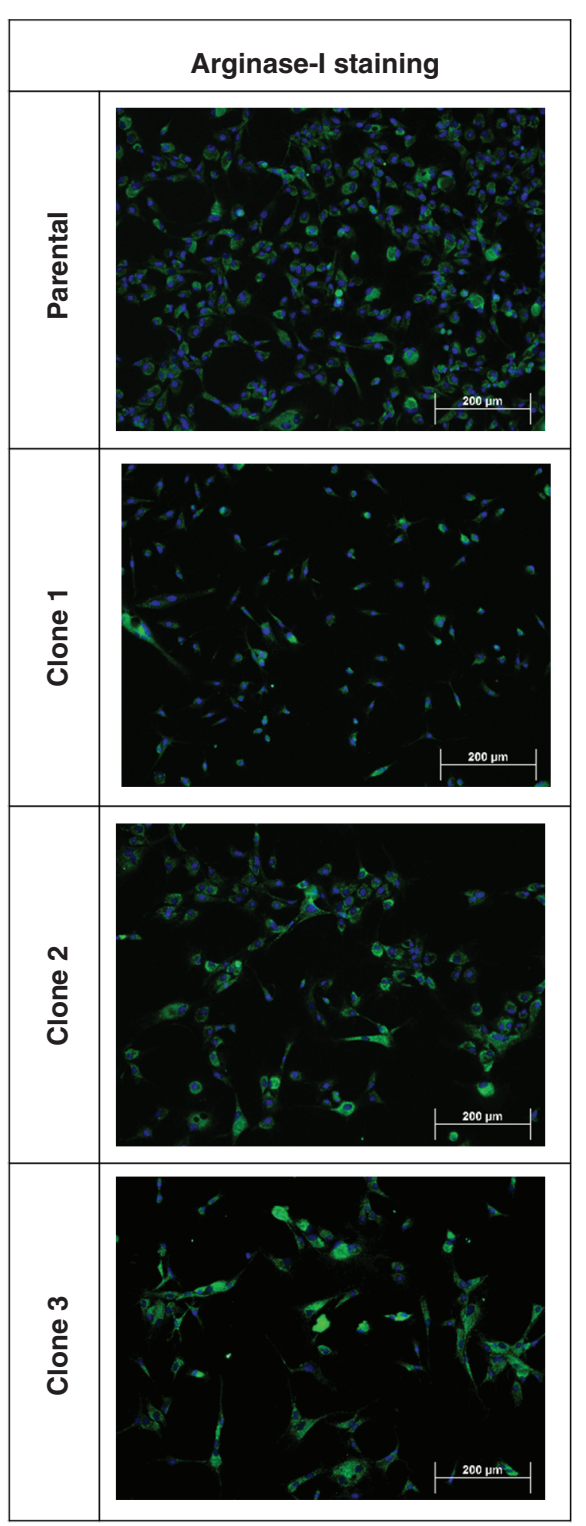

Figure 7. Representative single cell clones isolated from porcine hepatocellular carcinoma cells subject to CRISPR/Cas9-mediated ARID1A disruption. (A) Possible editing outcomes of CRISPR/Cas9 include the following: an identical indel is introduced in both alleles (homozygous mutation), different indels occur in each allele (biallelic mutation) or an indel is observed in an allele while the other allele remains unedited (heterozygous mutation). (B-D) Analysis of representative single cells clones isolated from porcine HCC cells transfected with Cas9 and ARID1A gRNA\#1. (B) Reads detected by targeted NGS analysis mapped to the reference sequence (top) for three single cell clones. Dashed line: predicted Cas 9 cleavage position; red box: insertion; dash: deleted base. (C) The predicted translation of ARID1A protein for the representative clones. The dotted region represents amino acids with frameshift mutation. (D) Arginase-1 staining (green) merged with DAPI staining (blue) for parental porcine HCC cell line and the representative single-cell clones (scale bar $=200 \mu \mathrm{m}$ ). All clones show positive arginase- 1 staining, confirming their hepatocellular origin. HCC: Hepatocellular carcinoma; NGS: Next-generation sequencing.

available antibodies are validated for human or mouse proteins. Another important consideration when performing CRISPR editing experiments is the assessment of off-target effects. These can be analyzed on a genetic level by deep sequencing of the top ten predicted off-target sites to confirm the presence or absence of off-target editing. Finally, while cell lines with the same intended gene knockout are expected to display similar phenotypes, clonal variation is commonly observed in gene editing experiments. Therefore, validation should be confirmed on a biological level by generating two knockout cell lines using two independent guides, followed by comparison of the 


\begin{tabular}{|c|c|c|c|c|c|}
\hline \multirow{6}{*}{ 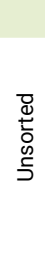 } & \multirow{2}{*}{$\begin{array}{l}\text { Clone } \\
\text { Clone } 1\end{array}$} & \multirow{2}{*}{$\begin{array}{l}\text { Allele } 1 \\
+1\end{array}$} & \multirow{2}{*}{$\begin{array}{l}\text { Allele } 2 \\
+1\end{array}$} & \multirow{2}{*}{$\begin{array}{l}\text { Editing outcome } \\
\text { Homozygous mutation }\end{array}$} & \multirow{2}{*}{$\begin{array}{l}\text { Knockout } \\
\text { Yes }\end{array}$} \\
\hline & & & & & \\
\hline & Clone 2 & -5 & -7 & Biallelic mutation & Yes \\
\hline & Clone 3 & -10 & No mutation & Heterozygous mutation & No \\
\hline & Clone 4 & +1 & -3 & Biallelic mutation & No \\
\hline & Clone 5 & No mutation & No mutation & No mutation & No \\
\hline \multirow{9}{*}{ 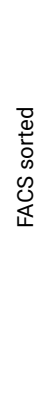 } & Clone 6 & -1 & -1 & Homozygous mutation & Yes \\
\hline & Clone 7 & -11 & -11 & Homozygous mutation & Yes \\
\hline & Clone 8 & -43 & -43 & Homozygous mutation & Yes \\
\hline & Clone 9 & -27 & -27 & Homozygous mutation & No \\
\hline & Clone 10 & -1 & +1 & Biallelic mutation & Yes \\
\hline & Clone 11 & -1 & -2 & Biallelic mutation & Yes \\
\hline & Clone 12 & -22 & +1 & Biallelic mutation & Yes \\
\hline & Clone 13 & -6 & +1 & Biallelic mutation & No \\
\hline & Clone 14 & -27 & -3 & Biallelic mutation & No \\
\hline
\end{tabular}

cellular phenotypes. This approach confirms that the resulting phenotypic changes are due to the gene knockout rather than unintended off-target perturbations.

The ability to precisely modify animal genes is highly valuable for functional investigation of disease-related mutations and for the generation of models that faithfully recapitulate human diseases. Here we present an approach to generating porcine HCC cells with tailored mutational profiles using CRISPR/Cas9 editing. By selecting a more efficient gRNA that targets our gene of interest and optimizing the transfection of RNP comprising Cas9 and gRNA, we achieved high editing efficiency of the porcine gene and isolated pure genetically validated knockout clonal populations.

\section{Future perspective}

Efficient utilization of the CRISPR/Cas system to edit the genome holds great promise for biomedical modeling. Our approach is based on in vitro editing of cells followed by orthotopic implantation to generate genetically tailored tumors. This approach can be expanded to generate porcine models of other types of cancers. The pipeline can also be applied in other species and can utilize human gene-edited cells in combination with immunocompromised animals. By optimizing the delivery of CRISPR components, in vivo editing of porcine genes can be used to generate models of various diseases. Such advances in generating animal models harboring customizable genetic mutations will expand the development and testing of precision medicine approaches.

Beyond the use of CRISPR/Cas to generate disease models, an extremely important benefit of optimizing and extensively testing this system in pigs is the ability to test novel therapeutics based on CRISPR in pigs prior to human clinical trials. Although CRISPR/Cas holds great promise in the therapy of genetic disorders by correcting disease-related mutations, several challenges impede its rapid application in human patients. Expanded testing of CRISPR as a therapeutic technique in animal models will lead to improvement of delivery efficiency, gene editing efficiency and toxicity assessment of the CRISPR/Cas9 system. Due to size, anatomical, and biological resemblance to humans, pigs represent an attractive animal model to test the safe and efficient use of the CRISPR/Cas 9 in humans.

\section{Executive summary}

- Porcine hepatocellular carcinoma $(\mathrm{HCC})$ cells were developed from transgenic pigs by inducing the expression of $T P 53^{R 167 H}$ and $K R A S^{G 12 D}$ transgenes.

- Mutations in the ARID1A gene were introduced in the porcine HCC cells by ribonucleoprotein delivery of CRISPR components.

- Selection of an optimal guide and enhancing the transfection efficiency resulted in $>90 \%$ in vitro editing of ARID1A in the porcine HCC cells.

Homogenous populations of ARID1A knockout cells were isolated, screened and validated.

\section{Author contributions}

R Gaba, K Schachtschneider and L Schook conceived the project. L Elkhadragy, M Regan, W Totura, K Dasteh Goli, S Patel, K Garcia and $M$ Stewart performed the experiments. L Elkhadragy and $M$ Regan analyzed and interpreted the data. L Elkhadragy wrote the manuscript. $\mathrm{R}$ Gaba and $\mathrm{K}$ Schachtschneider received funding. All the authors revised and edited the manuscript. 


\section{Acknowledgments}

The authors acknowledge the services provided by the Flow Cytometry Core, the Genome Research Core and the Fluorescence Imaging Core at the University of Illinois at Chicago.

\section{Financial \& competing interests disclosure}

This work was supported by the National Institutes of Health - National Cancer Institute (1R21CA219461-01A1), US Department of Defense (Translational Team Science Award CA150590) and the Department of Radiology, University of Illinois at Chicago. L Schook, R Gaba and K Schachtschneider have received research support from Guerbet USA LLC, Janssen Research \& Development LLC, the US Department of Defense and the US National Institutes of Health, and are scientific consultants for Sus Clinicals, Inc. L Elkhadragy, M Regan, W Totura, K Dasteh Goli, S Patel, K Garcia and M Stewart do not have conflicts of interest. The authors have no other relevant affiliations or financial involvement with any organization or entity with a financial interest in or financial conflict with the subject matter or materials discussed in the manuscript apart from those disclosed.

No writing assistance was utilized in the production of this manuscript.

\section{Ethical conduct of research}

All animal procedures were approved by the Institutional Animal Care and Use Committee at the University of Illinois at Chicago.

\section{Open access}

This work is licensed under the Attribution-NonCommercial-NoDerivatives 4.0 Unported License. To view a copy of this license, visit http: //creativecommons.org/licenses/by-nc-nd/4.0/

\section{References}

1. Flisikowska T, Kind A, Schnieke A. Pigs as models of human cancers. Theriogenology 86(1), 433-437 (2016)

2. Gun G, Kues WA. Current progress of genetically engineered pig models for biomedical research. Biores. Open Access 3(6), 255-264 (2014).

3. Hryhorowicz M, Lipinski D, Hryhorowicz S, Nowak-Terpilowska A, Ryczek N, Zeyland J. Application of genetically engineered pigs in biomedical research. Genes (Basel) 11 (6), 670 (2020).

4. Schachtschneider KM, Schwind RM, Darfour-Oduro KA et al. A validated, transitional and translational porcine model of hepatocellular carcinoma. Oncotarget 8(38), 63620-63634 (2017).

5. Gaba RC, Elkhadragy L, Boas FE et al. Development and comprehensive characterization of porcine hepatocellular carcinoma for translational liver cancer investigation. Oncotarget 11 , 2686-2701 (2020).

6. Yang JD, Hainaut P, Gores GJ, Amadou A, Plymoth A, Roberts LR. A global view of hepatocellular carcinoma: trends, risk, prevention and management. Nat. Rev. Gastroenterol. Hepatol. 16(10), 589-604 (2019).

7. Schook LB, Collares TV, Hu W et al. A genetic porcine model of cancer. PLoS ONE 10(7), e0128864 (2015).

8. Hsu PD, Lander ES, Zhang F. Development and applications of CRISPR-Cas9 for genome engineering. Cell 157(6), 1262-1278 (2014).

9. Yang L, Guell M, Niu D et al. Genome-wide inactivation of porcine endogenous retroviruses (PERVs). Science 350(6264), 1101-1104 (2015).

10. Wu JN, Roberts CW. ARID1A mutations in cancer: another epigenetic tumor suppressor? Cancer Discov. 3(1), 35-43 (2013).

11. Mathur R. ARID1A loss in cancer: towards a mechanistic understanding. Pharmacol. Ther. 190, 15-23 (2018).

12. Concordet JP, Haeussler M. CRISPOR: intuitive guide selection for CRISPR/Cas9 genome editing experiments and screens. Nucleic Acids Res. 46(W1), W242-W245 (2018).

13. Pinello L, Canver MC, Hoban MD et al. Analyzing CRISPR genome-editing experiments with CRISPResso. Nat. Biotechnol. 34(7), 695-697 (2016).

14. Chakrabarti AM, Henser-Brownhill T, Monserrat J, Poetsch AR, Luscombe NM, Scaffidi P. Target-specific precision of CRISPR-mediated genome editing. Mol. Cell 73(4), 699-713.e6 (2019).

15. Kouranova E, Forbes $\mathrm{K}$, Zhao $\mathrm{G}$ et al. CRISPRs for optimal targeting: delivery of CRISPR components as DNA, RNA, and protein into cultured cells and single-cell embryos. Hum. Gene Ther. 27(6), 464-475 (2016).

16. Kim S, Kim D, Cho SW, Kim J, Kim JS. Highly efficient RNA-guided genome editing in human cells via delivery of purified Cas9 ribonucleoproteins. Genome Res. 24(6), 1012-1019 (2014)

17. Zuris JA, Thompson DB, Shu Y et al. Cationic lipid-mediated delivery of proteins enables efficient protein-based genome editing in vitro and in vivo. Nat. Biotechnol. 33(1), 73-80 (2015).

18. Van Overbeek M, Capurso D, Carter MM et al. DNA repair profiling reveals nonrandom outcomes at Cas9-mediated breaks. Mol. Cell 63(4), 633-646 (2016). 